\title{
O EFEITO DE TIPOS DE BRINQUEDOS SOBRE O USO SIMPLES E COMBINADO DE ESTÍMULOS EM CRIANÇAS PRE-ESCOLARES*
}

Carmen Lúcia HUSSEIN F.M.U.
Edda BOMTEMPO

Instituto de Psicología da U.S.P.

- Trabalho perclalmente subvencionado pela C.A.P.E.S. A coleta de dados fol felta para a dissertaçăo de mestrado de Hussein; os dados, porém, foram tratados quando esta realizou um trabalho de curso na disciplina "Psicologia do Brinquedo II" curso de pós-graduaçáo do I.P.U.S.P., ministrada por Bomtempo, em 1980. 


\section{R E S U M O}

Este estudo insere-se na área do controle exercido pelo brinquedo sobre o comportamento. Usaram-se 16 crianças com idade média de 6 anos e nível sócio-econômico médio. Estas foram distribuídas em duas situações de mudança de composição sexual (seqüências $A B$ e BA). Como medida utilizou-se o uso simples e combinado de brinquedos apresentados em duplas. Foram usadas seis duplas de brinquedos, sendo que cada dupla era apresentada por dez minutos aos sujeitos, que foram observados aos pares. Os resultados foram os seguintes: a) os brinquedos da dupla foram mais usados um por vez do que combinadamente; b) o desempenho quanto ao uso combinado de estímulos face às diferentes duplas não apresentou diferenças significantes; e c) a mudança da composiçăo sexual parece năo ter sido relevante no controle dessas respostas. 
Vários autores mostram que o brinquedo como estímulo pode controlar determinadas respostas (Quilitch e Risley, 1972 e 1973; Hamad, Jackson e Risley, 1975; e Hussein, 1978).

Quilitch (1974) afirma que a avaliação do efeito de tipos de brinquedos sobre o comportamento é uma área que necessita de pesquisas experimentais, devido à importância do brinquedo para a criança préescolar e o seu possível uso no enriquecimento do repertório desta.

Assim, nessa área, encontraram-se vários estudos na década de trinta, como os de Updegraff e Herbst (1933), Parten (1933) e Johnson (1935). E também houve um ressurgimento de interesse por este tipo de questăo, na década de setenta, liderado principalmente por um grupo de pesquisadores da Universidade de Kansas, como os trabalhos de Quilitch e Risley, 1972 e 1973; Quilitch, Christophersen e Risley, 1977 e Quilitch, O'Brien e Risley, 1977, ligados a um projeto de descrição. seleçăo e organização de facilidades, equipamentos, materials e pessoas, além da intervenção usual sobre o comportamento.

o comportamento de brincar tem sido relacionado a habilidades em várias tarefas criativas. Assim, Lieberman (1965) encontrou relação slgnificante entre estes comportamentos em crianças de cinco anos e meio.

Vários autores têm usado como medidas de criatividade a fluêncla ideacional, a flexibilidade espontânea e a originalidade (Getzels e Jackson, 1962; Lieberman, 1965).

Sutton-Smith (1968) afirma que, quando uma criança brinca com um determinado estímulo, aumenta a extensão de associaçōes para aquele determinado objeto assim como varia as suas respostas e descobre vários usos para aqueles objetos. Além disso, alguns desses usos podem ser únicos. Esse autor conclul que o brinquedo pode fornecer experiências de enriquecimento do repertório da criança, que aprende a combinar $\theta$ associar estímulos para obter um resultado novo.

Nesse sentido, o trabaiho ora relatado teve como objetivos:

1. verificar o efeito de tipos de brinquedos sobre o uso simples e combinado de estímulos apresentados em duplas;

2. verificar o efeito das duplas de brinquedos sobre a utilização combinada de estímulos da dupla;

3. verificar o efeito da mudança da composição sexual (seqüências $A B$ e $B A]$ no controle destas respostas.

\section{MÉTODO}

\section{Sujeltos:}

16 crianças (metade de cada sexo) pré-escolares, matriculadas numa escola municipal de São Paulo, foram os sujeitos deste trabalho. 
A idade média dos sujeitos era de 72 meses e 13 dias e, quanto ao nível sócio-econômico, de acordo com a escala de Hutchinson (1960), os pais das crianças eram pertencentes à classe média, apresentando resultados que variavam de 14 a 23 pontos.

Os sujeitos foram distribuídos em duas situações $A B$ e $\mathrm{BA}$. $\mathrm{Na}$ situação $A B$, quatro pares (metade de cada sexo) foram submetidos primeiro a uma situação de homogeneidade por sexo (A) e depols redistribuídos em quatro pares mistos, passando por uma situaçaão de heterogeneidade por sexo (B). Na situação BA, outros quatro pares de crianças passaram por uma situaçăo inversa.

Material:

Os brinquedos foram estabelecidos através de pré-pesquisa.

Foram usados os seguintes brinquedos:

1) Trem de madeira (T) - "Trenzoca", da Fábrica de Brinquedos Saxônia, modelo D-302;

2) Blocos de construção e encaixe $\left(B_{1}\right)$ - "Blocos Criativos", da Fábrica de Brinquedos Trol, n. ${ }^{\circ}$ 35-07-0:

3) Blocos de construção $\left(\mathrm{B}_{2}\right)$ - "Car-Blocos", da Fábrica de Brinquedos Coluna, n. 5321;

4) Blocos de construçẫo $\left(B_{3}\right)-$ "O Futuro Engenheiro", da Fábrica de Brinquedos Coluna, n. ${ }^{\circ}$ 530-4;

5) Pega-Varetas (V), da Fábrica de Brinquedos Coluna, n. ${ }^{\circ} 1108$; 10.19.35;

6) Boneca Molly (Bo), da Fábrica de Brinquedos Estrela, n.

7) Baralho de animals (Ba) - "Primeiras Palavras - Sítio", da Fábrica de Brinquedos Grow, código 78v;

8) Caminhăo Betoneira (C), da Fábrica de Brinquedos Estrela, n. 20.05.14;

9) Qurebra-Cabeça $\left(O_{1}\right)$ - "O que é... O que é...", da Fábrica Grow, código 73v;

10] Quebra-cabeça em forma de cubos $\left(Q_{2}\right)-$ "Cubós Walt Disney", da Fábrica Coluna, n. 5349 ;

11) Blocos de construçāo e encaixe $\left(B_{4}\right)$ - "Blocos para construir - Polly", da Fábrìca Estrela, n. 20.27.05; 5337.

12) Dominó (D) - "Dominó de Figuras", da Fắbrica Coluna, n.

\section{Procedimento:}

Os sujeitos foram observados em uma sala que era usada como biblioteca da escola.

Duas sessōes experimentais, de trinta minutos cada uma, foram realizadas. A dupla de brinquedos que estava sobre a mesa foi trocada por outra (guardada numa sacola de plástico) a cada dez minutos.

Foram sorteadas pela tabela de números eqüiprováveis as duplas de brinquedos e a ordem de apresentação, que foram: 
1. dupla: Trenzoca (T) e Blocos Criativos $\left(B_{1}\right)$;

2. dupla: Car-Blocos $\left(B_{2}\right)$ e $O$ Futuro Engenheiro $\left(B_{3}\right)$;

3. dupla: Pega-Varetas (V) e Boneca Molly (Bo);

4. dupla: Primeiras Palavras (Ba) e Caminhão (C);

5." dupla: $O$ que é... $O$ que é... $\left(Q_{1}\right)$ e Cubos Walt Disney $\left(Q_{2}\right)$;

6.' dupla: Polly $\left(B_{4}\right)$ e Dominó de Figuras (D).

A primeira, a segunda e a terceira duplas foram apresentadas na primeira sessão; e a quarta, a quinta e a sexta duplas, na segunda sessão.

Um par de crianças por vez era trazido à sala e instruídas a brincar do jeito que quisessem, mas sempre com os brinquedos em cima da mesa. O experimentador registrava os estímulos $\mathrm{e}$ as categorias de respostas emitldas pelos sujeitos.

A técnica de observaçăo usada fol a amostra de tempo (time sampling).

A partir de pesquisas preliminares estabeleceu-se como definição de Resposta Simples (R. Si.) - quando duas crianças manipulam apenas um brinquedo da dupla apresentada, com todos os seus elementos, ou parte deles; e de Resposta Combinada (R. C.) - quando duas crianças manipulam os dois estímulos da dupla de brinquedos, com todos os seus elementos, ou parte deles.

A fidedignidade de registro dos estímulos e das categorias de respostas foi avaliada por dois observadores independentes. A concordâncla geral para as categorias de respostas foi de $97 \%$ e para os estímulos foi de $94 \%$.

Resultados:

Foi feita uma análise intra-grupos dos dados colhidos, considerando-se a categoria de uso simples e uso combinado de brinquedos.

Para isto, usou-se o teste de Wilcoxon (Siegel, 1956), indicado para amostras dependentes entre si e porque não se pode pressupor uma distribuição normal.

Primeiramente, comparou-se o uso de apenas um estímulo da dupla com o uso combinado de brinquedos da dupla. Admitiu-se como hipótese nula $\left(\mathrm{H}_{0}\right)$ que o desempenho no uso de um estímulo (R. Si.] seria igual ao uso combinado (R. C.) de dols brinquedos face a cada dupla de estímulos. Portanto, $\mathrm{H}_{0}$ : R. Si. $=$ R. C.. Como hipótese alternativa $\left(H_{1}\right)$, que o desempenho destas respostas seria diferente para cada dupla de brinquedos. Logo, $\mathrm{H}_{1}$ : R. Si. $\neq$ R. C.. O teste feito foi bicaudal.

A Tabela I mostrou que, na situaçăo $A B$, a hipótese nula foi rejeltada para $66,6 \%$ das comparações $e$, na situaçăo $B A$, a hip5tese nula foi rejeftada em cerca de $\mathbf{5 8 , 3} \%$ dos casos. O restante não apresentou diferenças significantes. Portanto, houve uma coerência dos dados quanto às duas situaçōes de composiçăo sexual, o que talvez indi- 
que que a seqüência parece năo ter sido relevante no uso dos estímulos, de modo simples ou combinadamente. Também se verificou uma tendência de serem os brinquedos usados um por vez do que combinadamente. Ainda, observou-se que os estímulos usados de modo simples foram os mesmos para as duas situaçōes, que foram: Trenzoca da dupla $\mathrm{TB}_{1}$, Blocos Criativos da dupla $\mathrm{TB}_{1}$, Baralho da dupla $\mathrm{BaC}$, O que é... O que é... da dupla $Q_{1} Q_{2}$, Cubos Walt Disney da dupla $\mathrm{Q}_{1} \mathrm{Q}_{2}$, Polly da dupla $\mathrm{B}_{4} \mathrm{D}$, e Dominó de Figuras da dupla $\mathrm{B}_{4} \mathrm{D}$.

\section{TABELA I}

Comparação entre o uso simples (R.Si.) e combinado (R.C.) de estímulos face a cada dupla de brinquedos nas situações AB e BA (significante ao nível 0,05 )

\begin{tabular}{|c|c|c|c|c|c|c|c|}
\hline \multicolumn{3}{|c|}{ Comparação } & \multirow{2}{*}{$\begin{array}{c}\text { Situação } \\
A B \\
\text { BA }\end{array}$} & \multirow{2}{*}{$\begin{array}{l}N \\
7 \\
8\end{array}$} & \multirow{2}{*}{$\begin{array}{l}\text { Tc } \\
2 \\
4\end{array}$} & \multirow{2}{*}{$\begin{array}{l}\text { To } \\
0 \\
2\end{array}$} & \multirow{2}{*}{$\begin{array}{l}\mathrm{H}_{0}: \text { R.Si. }=\text { R.C. }{ }^{*} \\
\left(\mathrm{H}_{1}: \text { R.Si. } \neq \text { R.C. }\right) \\
\mathrm{H}_{0}: \text { Rejeitada } \\
\mathrm{H}_{0}: \text { Rejeitada }\end{array}$} \\
\hline $\mathrm{T}$ & vs & $\mathrm{TB}_{1}$ & & & & & \\
\hline$\overline{\mathrm{B}_{1}}$ & vs & $\mathrm{TB}_{1}$ & $\begin{array}{l}\mathrm{AB} \\
\mathrm{BA}\end{array}$ & $\begin{array}{l}8 \\
8\end{array}$ & $\begin{array}{l}4 \\
4\end{array}$ & $\begin{array}{l}1,5 \\
0\end{array}$ & $\begin{array}{l}\mathrm{H}_{0}: \text { Rejeitada } \\
\mathrm{H}_{0}: \text { Rejeitada }\end{array}$ \\
\hline$\overline{B_{2}}$ & vs & $\mathrm{B}_{2} \mathrm{~B}_{3}$ & $\begin{array}{l}\mathrm{AB} \\
\mathrm{BA}\end{array}$ & $\begin{array}{l}7 \\
8 \\
\end{array}$ & $\begin{array}{l}2 \\
0\end{array}$ & $\begin{array}{r}3 \\
15 \\
\end{array}$ & $\begin{array}{l}\mathrm{H}_{0}: \text { Não Rejeitada } \\
\mathrm{H}_{0}: \text { Não Rejeitada }\end{array}$ \\
\hline$\overline{\mathrm{B}_{3}}$ & vs & $\mathrm{B}_{2} \mathrm{~B}_{3}$ & $\begin{array}{l}\mathrm{AB} \\
\mathrm{BA}\end{array}$ & $\begin{array}{l}7 \\
6 \\
\end{array}$ & $\begin{array}{l}4 \\
0\end{array}$ & $\begin{array}{r}9 \\
16\end{array}$ & $\begin{array}{l}\mathrm{H}_{0} \text { : Não Rejeitada } \\
\mathrm{H}_{0} \text { : Não Rejeitada }\end{array}$ \\
\hline$\overline{B o}$ & VS & BoV & $\begin{array}{l}\mathrm{AB} \\
\mathrm{BA}\end{array}$ & $\begin{array}{l}5 \\
6\end{array}$ & $\begin{array}{l}\text { Cá } \\
0\end{array}$ & $\begin{array}{c}\text { culo } \\
8\end{array}$ & $\begin{array}{l}\text { ossível } \\
\mathrm{H}_{0} \text { : Não Rejeitada }\end{array}$ \\
\hline$\overline{\mathrm{V}}$ & VS & BoV & $\begin{array}{l}\mathrm{AB} \\
\mathrm{BA}\end{array}$ & $\begin{array}{l}7 \\
7 \\
\end{array}$ & $\begin{array}{l}2 \\
2 \\
\end{array}$ & $\begin{array}{l}2 \\
3 \\
\end{array}$ & $\begin{array}{l}\mathrm{H}_{0}: \text { Rejeitada } \\
\mathrm{H}_{0}: \text { Não Rejeitada }\end{array}$ \\
\hline $\mathrm{C}$ & vs & $\mathrm{BaC}$ & $\begin{array}{l}\mathrm{AB} \\
\mathrm{BA}\end{array}$ & $\begin{array}{l}5 \\
7\end{array}$ & $\begin{array}{l}\text { Cá } \\
2\end{array}$ & $\begin{array}{l}\text { culo } \\
2,5\end{array}$ & $\begin{array}{l}\text { ossível } \\
\mathrm{H}_{0} \text { : Não Rejeitada }\end{array}$ \\
\hline$\overline{\mathrm{Ba}}$ & vs & $\mathrm{BaC}$ & $\begin{array}{l}\text { AB } \\
\text { BA }\end{array}$ & $\begin{array}{l}7 \\
8\end{array}$ & $\begin{array}{l}2 \\
4 \\
\end{array}$ & $\begin{array}{l}0 \\
0\end{array}$ & $\begin{array}{l}\mathrm{H}_{0}: \text { Rejeitada } \\
\mathrm{H}_{0}: \text { Rejeitada }\end{array}$ \\
\hline$\overline{\mathrm{Q}_{1}}$ & vs & $\mathrm{Q}_{1} \mathrm{Q}_{2}$ & $\begin{array}{l}\mathrm{AB} \\
\mathrm{BA}\end{array}$ & $\begin{array}{l}7 \\
8\end{array}$ & $\begin{array}{l}2 \\
4 \\
\end{array}$ & $\begin{array}{l}0 \\
0\end{array}$ & $\begin{array}{l}\mathrm{H}_{0}: \text { Rejeitada } \\
\mathrm{H}_{0}: \text { Rejeitada }\end{array}$ \\
\hline$\overline{\mathrm{Q}_{2}}$ & vs & $\mathrm{Q}_{1} \mathrm{Q}_{2}$ & $\begin{array}{l}\mathrm{AB} \\
\mathrm{BA}\end{array}$ & $\begin{array}{l}7 \\
7\end{array}$ & $\begin{array}{l}2 \\
2 \\
\end{array}$ & $\begin{array}{l}0 \\
2\end{array}$ & $\begin{array}{l}\mathrm{H}_{0}: \text { Rejeitada } \\
\mathrm{H}_{0}: \text { Rejeitada }\end{array}$ \\
\hline$\overline{\mathrm{B}_{4}}$ & vs & $\mathrm{B}_{4} \mathrm{D}$ & $\begin{array}{l}\mathrm{AB} \\
\mathrm{BA}\end{array}$ & $\begin{array}{l}6 \\
8\end{array}$ & $\begin{array}{l}0 \\
4\end{array}$ & $\begin{array}{l}0 \\
0\end{array}$ & $\begin{array}{l}\mathrm{H}_{0}: \text { Rejeitada } \\
\mathrm{H}_{0}: \text { Rejeitada }\end{array}$ \\
\hline $\bar{D}$ & vs & $\mathrm{B}_{4} \mathrm{D}$ & $\begin{array}{l}\mathrm{AB} \\
\mathrm{BA}\end{array}$ & $\begin{array}{l}6 \\
7\end{array}$ & $\begin{array}{l}0 \\
2\end{array}$ & $\begin{array}{l}0 \\
0\end{array}$ & $\begin{array}{l}\mathrm{H}_{0}: \text { Rejeitada } \\
\mathrm{H}_{0}: \text { Rejeitada }\end{array}$ \\
\hline
\end{tabular}

"R. Si. = o uso de apenas um estímulo de cada dupla de brinquedos;

R. C. = o uso de dois brinquedos da dupla. 
A seguir. comparou-se o total de R. Si. e R. C. face a todas as duplas. Temos como hipótese nula $\left(\mathrm{H}_{0}\right)$ que o desempenho das crianças seria igual quanto à utilização simples e combinada de estímulos face às seis duplas de brinquedos. Assim, $\mathrm{H}_{0}:$ T. R. Si. = T. R. C.. Como hipótese alternativa $\left(H_{\ell}\right)$ temos que os sujeitos apresentariam um desempenho diferentes nestas respostas face a todas as duplas de brinquedos. Logo, $H_{1}:$ T. R. SI. $\neq$ T. R. C.. Foi bicaudal o teste utilizado.

A Tabela II mostrou que a hipótese nula foil rejeitada para as seqüênciás $A B$ e $B A$, indicando que as crianças apresentaram mais uma tendência de usar os estímulos um por vez, frente a todas as duplas, do que combinadamente.

\section{TABELA II}

Comparação entre os totais de uso simples e combinado face a todas as duplas de brinquedos nas situações $A B$ e $B A$

(significante ao nível $\mathbf{0 , 0 5}$ )

\begin{tabular}{lcccccc}
\hline \multicolumn{2}{c}{ Comparação } & Situação & N & Tc & To & $\begin{array}{c}\mathrm{H}_{0}: \text { T.R.Si. }=\text { T.R.C. } \\
\left(\mathrm{H}_{1}: \text { T.R.Si } \neq \text { T.R.C. }\right)\end{array}$ \\
\hline T.R.Si. vs T.R.C. & AB & 8 & 4 & 0 & $\begin{array}{l}\mathrm{H}_{0}: \text { Rejeitada } \\
\mathrm{H}_{0} \text { : Rejeitada }\end{array}$ \\
T.R.Si. vs T.R.C. & BA & 8 & 4 & 0 & $\mathrm{H}^{2}$ Rejtada \\
\hline
\end{tabular}

"T. R. Si. = total de uso de apenas um brinquedo face a todas as duplas;

T. R. C. = total de uso combinado de brinquedos face às seis duplas.

A seguir, foram comparadas as duplas de brinquedos quanto ao uso combinado de brinquedos. Como hipótese nula tivemos que o desempenho do uso combinado de brinquedos numa dupla seria igual ao desempenho do uso combinado de outra dupla. Temos que $H_{0}: B_{1} B_{2}=$ $B_{3} B_{4}$. A hipótese alternativa seria que o desempenho do uso combinado de brinquedos de uma dupla seria diferente ao desempenho de outra dupla. Logo, $H_{1}: B_{1} B_{2} \# B_{3} B_{4}$.

A Tabela III mostrou, quanto à situaçăo $\mathrm{AB}$, que $80 \%$ das comparaçōes apresentaram cálculos impossívels, indicando que ou desempenho dos sujeitos era exatamente o mesmo nas duplas. Observou-se que a hipótese nula năo foi rejeitada para o restante. Ainda quanto à situaçăo BA, obteve-se $46,6 \%$ de casos em que a hipótese nula năo foi rejeitada, $\mathbf{4 0} \%$ de cálculos impossiveis e $14 \%$ de comparaçōes em que a hipótese nula foi rejeitada. Pode-se concluir que houve uma coerência dos dados obtidos, havendo uma tendência de os sujeitos desempenharem de modo igual quanto ao uso combinado de estímulos face a diferentes duplas. Ainda, pode-se afirmar que a seqüência $\mathbf{A B}$ e BA não parece ter sido uma variável relevante no controle do uso combinado de brinquedos. 
TABELA III

Comparação entre as duplas de brinquedos quanto ao uso combinado nas sîtuaçōes $A B$ e $B A$ (significante ao nivel 0,05 )

\begin{tabular}{|c|c|c|c|c|c|}
\hline \multicolumn{3}{|c|}{ Comparação } & \multirow{2}{*}{$\begin{array}{c}\text { Situação } \\
\mathrm{AB} \\
\mathrm{BA}\end{array}$} & \multirow{2}{*}{$\begin{array}{l}N \\
7 \\
6\end{array}$} & \multirow{2}{*}{$\begin{aligned} & \mathrm{H}_{0}: \mathrm{B}_{1} \mathrm{~B}_{2}=\mathrm{B}_{3} \mathrm{~B}_{4}{ }^{*} \\
&\left(\mathrm{H}_{1}: \mathrm{B}_{1} \mathrm{~B}_{2}\right.\left.\neq \mathrm{B}_{3} \mathrm{~B}_{4}\right) \\
& \mathrm{H}_{0}: \text { Não Rejeitada } \\
& \mathrm{H}_{0}: \text { Não Rejeitada }\end{aligned}$} \\
\hline $\mathrm{TB}_{1}$ & vs & $\mathrm{B}_{2} \mathrm{~B}_{3}$ & & & \\
\hline$T B_{1}$ & vs & $\mathrm{BoV}$ & $\begin{array}{l}\mathrm{AB} \\
\mathrm{BA}\end{array}$ & $\begin{array}{l}6 \\
3\end{array}$ & $\begin{array}{l}0 \quad 11 \quad \mathrm{H}_{0}: \text { Năo Rejeitada } \\
\text { Cálculo Impossivel }\end{array}$ \\
\hline $\mathrm{TB}_{1}$ & vs & $\mathrm{CBa}$ & $\begin{array}{l}\mathrm{AB} \\
\mathrm{BA}\end{array}$ & $\begin{array}{l}5 \\
8\end{array}$ & $\begin{array}{l}\text { Cálculo Impossível } \\
4\end{array} 8,5 \quad H_{0}:$ Não Rejeitada \\
\hline $\mathrm{TB}_{1}$ & vs & $\mathrm{Q}_{1} \mathrm{O}_{2}$ & $\begin{array}{l}\mathrm{AB} \\
\mathrm{BA}\end{array}$ & $\begin{array}{l}5 \\
4 \\
\end{array}$ & $\begin{array}{l}\text { Cálculo Impossive! } \\
\text { Cálculo Impossível }\end{array}$ \\
\hline $\mathrm{TB}_{\mathrm{I}}$ & vs & $\mathrm{B}_{4} \mathrm{D}$ & $\begin{array}{l}\mathrm{AB} \\
\mathrm{BA}\end{array}$ & $\begin{array}{l}4 \\
2 \\
\end{array}$ & $\begin{array}{l}\text { Cálculo Impossivel } \\
\text { Cálculo Impossivel }\end{array}$ \\
\hline $\mathrm{B}_{2} \mathrm{~B}_{3}$ & vs & $\mathrm{BoV}$ & $\begin{array}{l}\mathrm{AB} \\
\mathrm{BA}\end{array}$ & $\begin{array}{l}3 \\
6\end{array}$ & $\begin{array}{lcc}\text { Cálculo impossível } & \\
0 & 4 & \mathrm{H}_{0}: \text { Não Rejeitada }\end{array}$ \\
\hline $\mathrm{B}_{2} \mathrm{~B}_{3}$ & vs & $\mathrm{CBa}$ & $\begin{array}{l}\mathrm{AB} \\
\mathrm{BA}\end{array}$ & $\begin{array}{l}3 \\
8\end{array}$ & 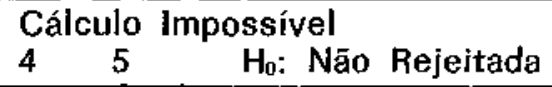 \\
\hline $\mathrm{B}_{2} \mathrm{~B}_{3}$ & vs & $\mathrm{Q}_{1} \mathrm{Q}_{2}$ & $\begin{array}{l}\mathrm{AB} \\
\mathrm{BA}\end{array}$ & $\begin{array}{l}5 \\
7\end{array}$ & $\begin{array}{l}\text { Cálculo lmpossivel } \\
2 \quad 2,5 \quad H_{0}: \text { Não Rejeitada }\end{array}$ \\
\hline$B_{2} B_{3}$ & vs & $\mathrm{B}_{4} \mathrm{D}$ & $\begin{array}{l}\mathrm{AB} \\
\mathrm{BA}\end{array}$ & $\begin{array}{l}3 \\
6\end{array}$ & $\begin{array}{l}\text { Cálculo Impossivel } \\
0 \quad 0 \quad H_{0}: \text { Rejeitada }\end{array}$ \\
\hline BoV & vs & $\mathrm{CBa}$ & $\begin{array}{l}\mathrm{AB} \\
\mathrm{BA}\end{array}$ & $\begin{array}{l}4 \\
7 \\
\end{array}$ & $\begin{array}{lll}\text { Cálculo Impossível } \\
2 & 12,5 & \mathrm{H}_{0} \text { : Não Rejeitada }\end{array}$ \\
\hline $\mathrm{BoV}$ & vs & $\mathrm{O}_{1} \mathrm{O}_{2}$ & $\begin{array}{l}\mathrm{AB} \\
\mathrm{BA}\end{array}$ & $\begin{array}{l}3 \\
5 \\
\end{array}$ & $\begin{array}{l}\text { Cálculo Impossivel } \\
\text { Cálculo Impossivel }\end{array}$ \\
\hline BoV & vs & $\mathrm{B}_{4} \mathrm{D}$ & $\begin{array}{l}\mathrm{AB} \\
\mathrm{BA}\end{array}$ & $\begin{array}{l}6 \\
3\end{array}$ & $\begin{array}{l}0 \\
0 \\
\text { Cálculo Impossível }\end{array}$ \\
\hline $\mathrm{CBa}$ & vs & $\mathrm{Q}_{1} \mathrm{Q}_{2}$ & $\begin{array}{l}\mathrm{AB} \\
\mathrm{BA}\end{array}$ & $\begin{array}{l}3 \\
8\end{array}$ & $\begin{array}{l}\text { Cálculo Impossível } \\
4 \quad 9,5 \quad H_{0}: \text { Não Rejeitada }\end{array}$ \\
\hline $\mathrm{CBa}$ & vs & $\mathrm{B}_{4} \overline{\mathrm{D}}$ & $\begin{array}{l}\mathrm{AB} \\
\mathrm{BA}\end{array}$ & $\begin{array}{l}3 \\
7 \\
\end{array}$ & \begin{tabular}{lcc}
\multicolumn{3}{l}{ Cálculo impossível } \\
2 & 0 & $\mathrm{H}_{0}:$ Rejeitada \\
\end{tabular} \\
\hline $\mathrm{O}_{1} \mathrm{Q}_{2}$ & vs & $B, D$ & $\begin{array}{l}\mathrm{AB} \\
\mathrm{BA}\end{array}$ & $\begin{array}{l}3 \\
3\end{array}$ & $\begin{array}{l}\text { Cálculo Impossível } \\
\text { Cálculo Impossivel }\end{array}$ \\
\hline
\end{tabular}

${ }^{-} \mathrm{B}_{1} \mathrm{~B}_{2}=$ uma das duplas de brinquedos comparadas:

$\mathrm{B}_{3} \mathrm{~B}_{4}=$ outra dupla de brinquedos. 


\section{Discussāo e Conclusões:}

Seguiu-se, na medida do possivel, a ordem dos objetivos.

Quanto ao primeiro objetivo, que foi $o$ de verificar o efeito de tipos de brinquedos sobre o uso simples e combinado de estímulos apresentados em duplas, encontrou-se, tanto para a situação $A B$ como para BA, uma tendência de serem estes usados um por vez do que combinadamente (Tabela 1).

Esta indicação foi confirmada no tratamento do total destas respostas, face às seis duplas, havendo portanto uma coerência entre estes dois tratamentos (Tabela II).

Uma questāo importante a ser discutida é em que medida as variáveis de estímulo e variáveis do sujeito afetariam estes resultados.

Quanto ao primeiro aspecto, o brinquedo como estímulo, o tipo de material empregado parece ter controlado estes resultados. No entanto, usou-se uma variaçāo pequena de brinquedos, quatro blocos e três quebra-cabeças, o que pode sugerir a necessidade de pesquisas posteriores usando uma diversidade maior de tipos de brinquedos.

Uma outra explicação para estes resultados é que cada brinquedo da dupla parece ter sido muito reforçador, acarretando talvez o controle do uso de apenas um estímulo e, possivelmente, a não procura do outro estímulo da dupla.

Assim, Hussein (1978), focalizando a interação social de crianças pré-escolares, verificou que estes mesmos brinquedos foram reforçadores e que levaram a uma maior emissão de resposta não social do que social. Ela discute esses dados considerando que esses sujeitos pareciam ter sido mais controlados pelo brinquedo do que pelo companheiro como estimulo.

Quanto à variável do sujeito, uma explicaçāo a ser dada é que os sujeitos deste trabalho nāo teriam desenvolvido um repertório adequado de flexibilidade de respostas em situaçāo de brinquedo.

Este fato talvez se deva a certas caracteristicas de seu ambiente sócio-educacional, que os levariam a não alterarem a estimulação dos brinquedos apresentados em dupla, não os usando combinadamente.

Assim, o tipo de treino possivelmente fornecido pela família e escola não possibilitaria o desenvolvimento desta habilidade. Além disso, Barnes (1971) sugere que as crianças atualmente estāo mais expostas à comunicação de massa e brincam menos, o que nos leva a supor que tenham menos oportunidade de aumentar a extensão de associação para determinados objetos, assim como pouca oportunidade de variar as suas respostas e dar muitos usos a estes.

Neste sentido, Pulanski (1970), num estudo de crianças de cinco a sete anos, observou que o efeito de dois conjuntos de brinquedos - muito e pouco estruturado - não foi tăo importante nesta idade e 
que a classificação dos sujeitos em alta e baixa predisposiçāo a fantasia foj a variável mais relevante no desenvolvimento de imaginação destes quando participam em jogo imaginativo. A autora discute estes dados em relaçăo à idade, afirmando que, nesta faixa de idade, a criança já tem bem estabelecida a predisposição a fantasia, o que a leva a não cosiderar as circunstâncias. Sugere a necessidade de estudos com crianças menores para determinar em que idade o tipo de brinquedo pode afetar o desenvolvimento de imaginação.

Seriam necessários estudos em que se medissem habilidades em várias tarefas criativas e fossem fornecidos diversos tipos de materiais a crianças pré-escolares de diferentes faixas etárias, para determinar em que idade ela começaria a desenvolver esta habilidade, assim como em que idade ela seria mais controlada pelo tipo de brinquedo.

Quanto ao segundo objetivo, que foi o de verificar o efeito das duplas de brinquedos sobre a utilização combinada de estímulos da dupla, verificou-se que houve um desempenho igual dos sujeitos, face a cada dupla, nas duas situações (Tabela III). Considerando-se isto e a indicaçāo de que os brinquedos foram usados mais isoladamente do que juntos, pode-se afirmar que houve uma coerência interna entre estes tratamentos.

O terceiro objetivo foi o de verificar o efeito da mudança de composição sexual (situações $A B$ e BA) sobre o uso simples e combinado de estímulos.

A variável mudança da composição sexual (situações $A B$ e $B A$ ) nāo pareceu ter sido relevante no controle destas respostas nos três tratamentos realizados (Tabelas 1 , II e III). Verificou-se que o desempenho dos sujeitos foi igual nas situaçóes $A B$ e BA. Os dados encontrados parecem servir de apoio às conclusōes de Pulanski (1970), Langlois et al, (1973) e Hussein (1978).

Pode-se concluir que são necessários outros estudos que controlem melhor as variáveis do sujeito, isto é, explicitando as habilidades desempenhadas em tarefas criativas, assim como usando outras medidas de criatividade na situaçăo de brinquedo, na medida em que este é um estímulo reforçador para a criança pré-escolar, é uma técnica de estudo do repertório cognítivo desta, assim como pode ser um instru. mento de treíno de respostas nesta área.

\section{REFERENCIAS BIBLIOGRAFICAS}

BARNES. K. E. - Preschool Play Norms: A replication. Developmental Psychology. 1971, 5(1):99-103.

GETZELS, J. W. E JACKSON, P. W. - Creativity and Intelligence. New York. Wiley. 1962.

HAMAD, C. D.; JACKSON, E. H. e RISLEY, T. R. - The selection of toys as the basis for maximlzing appropriate play behavior in chlidren. Paper presented at American Paychological Associetion. Chlcago, 1975. 
HUSSEIN, C. L. - o efelto da mudança de composição sexual em respostas de criancas pré-escolares face a duples de brinquedos. Tese de mestrado apresentada ao I.P.U.S.P.. São Paulo, 1978.

HUTCHINSON, B. - Mobilidade e Trabalho. Rio de Janeiro. Centro Brasilelro de Pesquisas Educacionals, 1960.

HUTT, S. J. e HUTT, C. - Observaçăo direta e medida do comportamento. Traduçāo do original ingles de 1970 por C. M. Bori. Sło Paulo. E.D.U.S.P., 1974.

JOHNSON. M. W. - The effect on behavior of variation in the amount of play equipment. Child Development, 1935, 6:56-68.

LANGLOIS, J. H.; GOTTFRIED, N. W. e SEAY, B. - The influence of sex of peer on the social behavior of preschool children. Developmental Psychology, 1973, 8(1):93-98.

LIEBERMAN, J. N. - Playfulness and Divergent Thinking: an investigation of their relationship at the kindergarten level. The Journal of Genetic Psychology, 1965, 107:219-224.

PARTEN, M. B. - Social Play among preschool children. (1933) in: R. G. Herron $\theta$. Sutton-Smith. Child's Play. New York. Wiley, 1971.

PULANSKI. M. A. S. - Play as a function of toy structure and fantasy predisposition. Child Development, 1970, 4t:531-537.

OUILITCH, H. R. - How Educatlonal are educational toys? Center for Applied Behavior Analysls. Lawrence, Kansas, 1974.

QUiLITCH, H. R.; CHRISTOPHERSEN, E. R. - RISLEY, T. R. - The evaluation of children's play materials. Jotumal of Applied Behavior Analysis, 1977, 10:501-502.

OUILITCH, H. R.; O'BRIEN, M. e RISLEY, T. R. - Evaluating Toys: Procedures and materials for conducting toys evaluations. Livlng Envlronments Group, Kansas, 1977.

QUILITCH, H. R. \& RISLEY, T. R. - The organization of group care environments: toy evaluation. Paper presented at American Psychological Assoclation, Honolulu, 1972.

OUILITCH. H. R. e RISLEY, T. R. - The effects of play material on social play. Journal of Applied Behavior Analysis, 1973, 6:573-578.

SIEGEL, S. - Non parametric statistics for the behavioral sclences. New York. McGraw Hill Book Co., 1956.

SUTTON-SMITH, B. - The role of play In Cognitive Development. Young children, 1967, 22:(6):361-370.

UPDEGRAFF. R. e HERBST, E. K. - An experimental study of the social behaviour stimulated in young chlldren by certain play materials. J. Genet. Psychol., 1933, 42:372-391. 